\title{
ShadowTutor: Distributed Partial Distillation for Mobile Video DNN Inference
}

\author{
Jae-Won Chung* \\ Seoul National University \\ Seoul, South Korea \\ jaywonchung@snu.ac.kr
}

\author{
Jae-Yun Kim \\ Seoul National University \\ Seoul, South Korea \\ jaeykim@altair.snu.ac.kr
}

\author{
Soo-Mook Moon* \\ Seoul National University \\ Seoul, South Korea \\ smoon@snu.ac.kr
}

\begin{abstract}
Following the recent success of deep neural networks (DNN) on video computer vision tasks, performing DNN inferences on videos that originate from mobile devices has gained practical significance. As such, previous approaches developed methods to offload DNN inference computations for images to cloud servers to manage the resource constraints of mobile devices. However, when it comes to video data, communicating information of every frame consumes excessive network bandwidth and renders the entire system susceptible to adverse network conditions such as congestion. Thus, in this work, we seek to exploit the temporal coherence between nearby frames of a video stream to mitigate network pressure. That is, we propose ShadowTutor, a distributed video DNN inference framework that reduces the number of network transmissions through intermittent knowledge distillation to a student model. Moreover, we update only a subset of the student's parameters, which we call partial distillation, to reduce the data size of each network transmission. Specifically, the server runs a large and general teacher model, and the mobile device only runs an extremely small but specialized student model. On sparsely selected key frames, the server partially trains the student model by targeting the teacher's response and sends the updated part to the mobile device. We investigate the effectiveness of ShadowTutor with HD video semantic segmentation. Evaluations show that network data transfer is reduced by $95 \%$ on average. Moreover, the throughput of the system is improved by over three times and shows robustness to changes in network bandwidth.
\end{abstract}

\section{CCS CONCEPTS}

- Human-centered computing $\rightarrow$ Mobile computing; • Computing methodologies $\rightarrow$ Distributed artificial intelligence; Distributed computing methodologies.

\section{KEYWORDS}

Deep neural networks, distributed inference, knowledge distillation, video semantic segmentation

"corresponding authors

Permission to make digital or hard copies of all or part of this work for personal or classroom use is granted without fee provided that copies are not made or distributed for profit or commercial advantage and that copies bear this notice and the full citation on the first page. Copyrights for components of this work owned by others than ACM must be honored. Abstracting with credit is permitted. To copy otherwise, or republish, to post on servers or to redistribute to lists, requires prior specific permission and/or a fee. Request permissions from permissions@acm.org.

ICPP '20, August 17-20, 2020, Edmonton, AB, Canada

(C) 2020 Association for Computing Machinery.

ACM ISBN 978-1-4503-8816-0/20/08 . .\$15.00

https://doi.org/10.1145/3404397.3404404
ACM Reference Format:

Jae-Won Chung, Jae-Yun Kim, and Soo-Mook Moon. 2020. ShadowTutor: Distributed Partial Distillation for Mobile Video DNN Inference. In 49th International Conference on Parallel Processing - ICPP (ICPP '20), August 17-20, 2020, Edmonton, AB, Canada. ACM, New York, NY, USA, 11 pages. https://doi.org/10.1145/3404397.3404404

\section{INTRODUCTION}

Deep learning approaches have proved to be extremely powerful in fields such as computer vision [13], natural language processing [29], speech recognition [4], and sequential recommendation [14]. Due to their applicability to various services, such DNN models sparked interest for performing DNN inference directly on data generated on mobile devices.

Video is one of the most important among such data due to its numerous practical applications. Autonomous vehicles perform road segmentation to traverse the street and detect obstacles [27]. CCTV cameras used for home security can notice movements and detect objects using DNNs [16]. Mobile selfie apps segment humans and the rest to change the background or simulate background blurring effects [3]. Thus, video DNN inferences on comparatively weak devices have become a core mechanism for delivering various services and technologies.

While running a large model directly on-device may yield high accuracy, execution latency and memory consumption can quickly become unacceptable for resource-constrained mobile devices. Thus, to lift the mobile device of the unwieldy inference workload, several approaches offload DNN computations to cloud servers [8, 12, 17]. In this scheme, the mobile device sends video frames to the cloud server and retrieves inference results. However, these approaches are limited in that information of every frame must be communicated between the server and the client. This heavily pressures network traffic, which is a view shared with [1]. Moreover, the entire system becomes vulnerable to adverse network conditions such as reduction in available network bandwidth due to congestion.

To this end, we propose ShadowTutor, a general video DNN inference framework. Here, a very small pre-trained student model runs on a mobile device for test time inference. However, due to its size, the student lacks generalization power to excel on any given input scene even if it has undergone "public education". Thus, to enhance performance specifically on the video at hand, the student receives "shadow education" from a large teacher model on the server. Especially, the student receives training only on a fraction of all frames in the video, which frames we call key frames.

The student model on the mobile device processes video frames one by one in an online manner. On sparse key frames, the mobile device sends the frame to the server. Upon key frame receive, the server updates a copy of the student by considering the teacher's 
inference result as the key frame's label, and returns the updated student weights to the mobile device. In effect, the student is specialized to the current video stream by distilling knowledge from the teacher during test time. Now, the student model can accurately perform inference on-device without the teacher's help for non-key frames, thanks to the temporal coherence between the key frame and the non-key frames after it. That is, non-key frames are likely to share characteristics such as background, ambience, overall texture, speed of movement, and the objects present with a nearby key frame, thus allowing the student trained on that key frame to accurately process non-key frames that come after it. Moreover, the mobile device does not wait until the updated student weights arrive; it just proceeds to the next non-key frame asynchronously with the slightly outdated student, thereby exploiting temporal coherence further. Finally, the stride to the next key frame is determined by a simple but adaptive algorithm based on the student's performance after distillation.

Sending the updated weights to the mobile device incurs little overhead because the student is very small by design. For instance, in our experiment, the student model is even smaller than a single video frame. Still, we propose partial distillation, a technique that further reduces the transmitted data size, and apply it to ShadowTutor. That is, upon distilling knowledge from the teacher, only a subset of the student's parameters is trained. This technique accelerates knowledge distillation and allows only the updated part of the student to be transmitted across the network. Moreover, surprisingly, partial distillation yields generally higher accuracy and further reduces the number of network communications compared with adapting all parameters.

We evaluate the effectiveness of ShadowTutor on HD video semantic segmentation, a challenging video computer vision task that requires dense predictions of pixel-level class probabilities. Evaluations show that compared with naive DNN offloading, ShadowTutor reduces network data transfer by $95 \%$ on average and improves throughput by over 3x. Also, due to asynchronous inference, ShadowTutor effectively retains throughput under low available network bandwidth.

In sum, we make the following contributions:

- We design ShadowTutor, a distributed video DNN inference framework that reduces the number of network transmissions and the data size of each.

- We allow ShadowTutor to be robust to changes in network conditions by adopting asynchronous inference.

- We aid the configuration of ShadowTutor by providing analytic models of its network traffic and throughput in terms of system parameters and component measurements.

- We adapt ShadowTutor to HD video semantic segmentation, a real-life application, and evaluate its performance in terms of throughput, network traffic, accuracy, and robustness.

The rest of this paper is organized as follows. We review previous approaches in section 2, and provide background regarding our work in section 3. We explain the core idea and mechanism of ShadowTutor in section 4, and show how it is adapted specifically to video semantic segmentation in section 5 . Then, we evaluate our framework in section 6. Finally, we introduce related work in section 7 and conclude the paper in section 8 .

\section{PREVIOUS APPROACHES}

Due to the resource constraints of mobile devices, DNN computations are often offloaded to central cloud servers. Neurosurgeon [17] partitions a given DNN and collaboratively executes it between the cloud and the server. It searches for an optimal partition point in terms of latency and power consumption. Eshratifar et al [8] also seeks to partition DNNs, but by deriving optimal conditions for client power consumption and cloud resource constraints. MCDNN [12] generates modified and specialized versions of the original model to trade-off accuracy for improved resource usage. However, it only deals with models that perform image classification, requires the execution of the model for every image in the training set for specialization (pre-forwarding), and the generated model is mostly limited to being a layer-wise modification of the original model. Moreover, adopting these approaches still require the communication of every frame across the network, thereby pressuring network traffic greatly.

Another work by Yang et al. [31] splits video frames into partitions that are distributed to slaves. It utilizes optical flow, i.e. the movement vectors between two frames, to exploit the temporal coherence between frames. However, their system still requires the communication of every single frame between the master and the slaves, since they utilize temporal coherence only to occasionally reduce the amount of computation, rather than reducing network traffic.

\section{BACKGROUND}

\subsection{Knowledge Distillation}

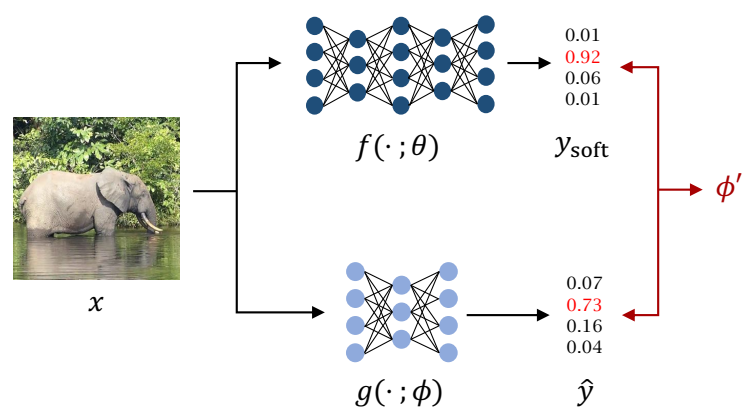

Figure 1: Inference time knowledge distillation. The student's parameters are updated by viewing the teacher's ouptut as the label.

Knowledge distillation [15] was initially proposed as a means of model compression. Viewing a large teacher model's output as a soft target, it formulated a knowledge distillation loss between the output of a small student model and the soft target. Compared with hard targets, which are one-hot vectors from the dataset, soft targets provide much more information about the input data, and allow less variance in the gradients of the student model.

Figure 1 illustrates the concept of knowledge distillation applied to image classification. Since it assumes inference time, the actual label $y_{\text {hard }}$ is not available. Thus, the student learns just from the output of the teacher model $y_{\text {soft }}$. Is is also the case for ShadowTutor. 


\subsection{Semantic Segmentation}

Semantic segmentation is in other words pixel-wise classification. There is a fixed set of classes, including the background class. Then, the model is required to classify each pixel of its input image to one of the given classes. Thus, the output of semantic segmentation has the same spatial size as the input image.

The result of semantic segmentation is often evaluated with mean IoU (Intersection over Union). With pred $_{c}$ the set of pixels classified as class $c$ and label $_{c}$ the set of pixels with ground truth class $c$, the IoU for class $c$ is defined as

$$
\mathrm{IoU}_{c}=\frac{\mid \text { pred }_{c} \cap \text { label }_{c} \mid}{\mid \text { pred }_{c} \cup \text { label }_{c} \mid} .
$$

The IoU is computed for each class in the ground truth label and averaged to yield the mean IoU performance metric. Mean IoU thus lies between 0 and 1 , with 1 being the best possible score.

\section{SHADOWTUTOR}

Figure 2 shows an overview of ShadowTutor. All frames are processed sequentially in temporal order. Determined by the key frame scheduling policy, if the current frame is a non-key frame, its inference is handled entirely on-device by a lightweight student model. If the current frame is a key frame, it is sent to the server. Using the key frame, the server updates the a copy of the student's weights via knowledge distillation from the teacher, and returns the updated weights to the client. Network communication occurs only at sparse key frames, allowing ShadowTutor to reduce the amount of network traffic drastically.

\subsection{System Components}

Stripping away the distributed nature from ShadowTutor, there are five essential components that comprise the system: video data, teacher inference, student inference, student training, and key frame striding.

4.1.1 Video data. Video data reside in the mobile device, and the majority of the frames do not leave its home. The video can either be in an internal storage or fetched from the camera in real-time. Since each frame is traversed by the system in strict temporal order without look-back, frames can be readily discarded once they are processed.

4.1.2 Teacher inference. By teacher, we refer to a heavy but highquality neural network deployed to the server. Since ShadowTutor is an inference system, labels for the input data do not exist. Thus, it is important to employ a sufficiently good teacher model that can generalize well to unseen video scenes. Also, the teacher should be pre-trained on a dataset relevant to the task. Note that teacher inference is done only for the key frames transmitted to the server.

4.1.3 Student inference. By student, we refer to a lightweight neural network designed to run on the mobile device. The student model need not be deployed to the mobile device when ShadowTutor is installed, because the server can simply supply the student weights when the system starts. The student should also be pre-trained on relevant data, most likely the data used to pre-train the teacher. Pre-training can be expensive, but it is a one-time cost.

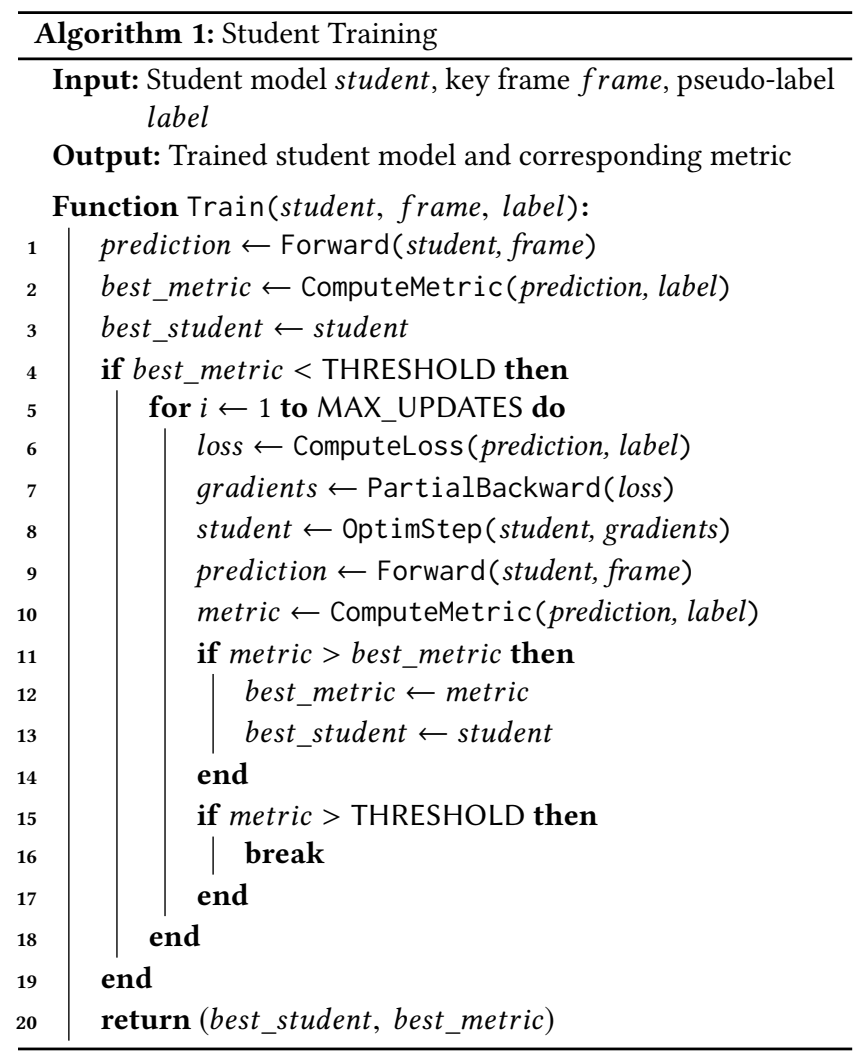

For non-key frames, student inference is the only operation done on them. Hence, student inference latency determines the steady state throughput of ShadowTutor.

4.1.4 Student training. With the teacher's inference result, deemed the pseudo-label, the student model is trained. Since the student model is very small, it lacks generalization power to perform well on any video stream it encounters. However, in our setting, there is no need for generalization; we only need to do well for the video at hand. Thus, by training the student with sparse key frames, we overfit the student model to the current video stream.

Algorithm 1 shows the process of student training. Optimization steps (most simply gradient descent) for the student are taken until either the performance metric (e.g. accuracy, mean IoU) exceeds THRESHOLD, or the number of training steps reach MAX_UPDATES. The best performing student and the corresponding metric is returned. THRESHOLD and MAX_UPDATES are algorithmic parameters. The former denotes an acceptable level of student performance, and the latter the maximum number of optimization steps. Especially, the higher the THRESHOLD, the more the expected number of optimization steps, since it becomes harder for the student to break out of the training loop. Thus, increasing either of the two potentially increases student performance, but the system's throughput decreases. Moreover, we perform partial distillation, as denoted by the function PartialBackward. We discuss this in greater detail in section 4.2 .

We emphasize that this process be distinguished from student pre-training; student training is done repetitively during system 


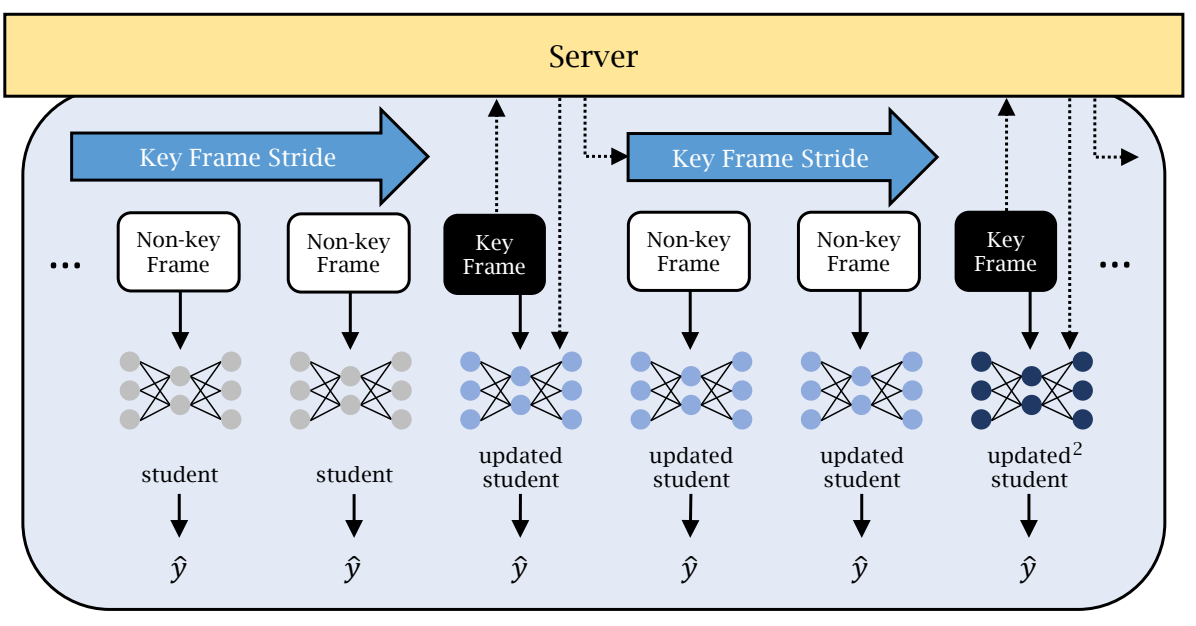

Client

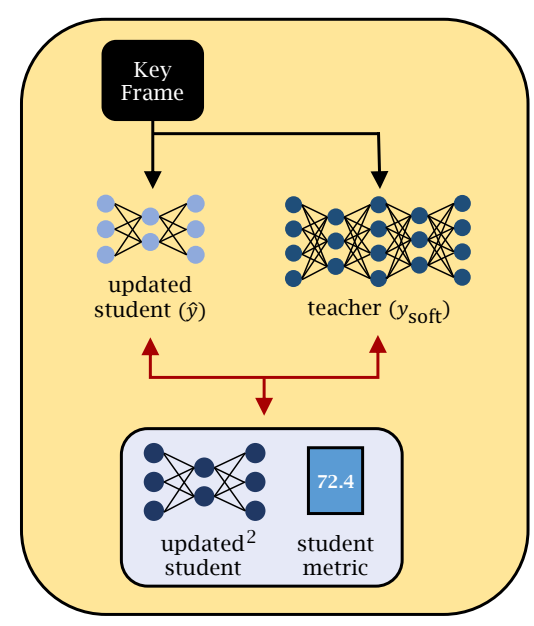

Server

Figure 2: High-level view of ShadowTutor. Dotted arrows denote network communication, while other arrows denote data transfers within a device. Each frame is processed one by one sequentially. Non-key frame inferences are done on-device with a small student network. Sparse key frames are used to update the student's weights via knowledge distillation from a teacher.

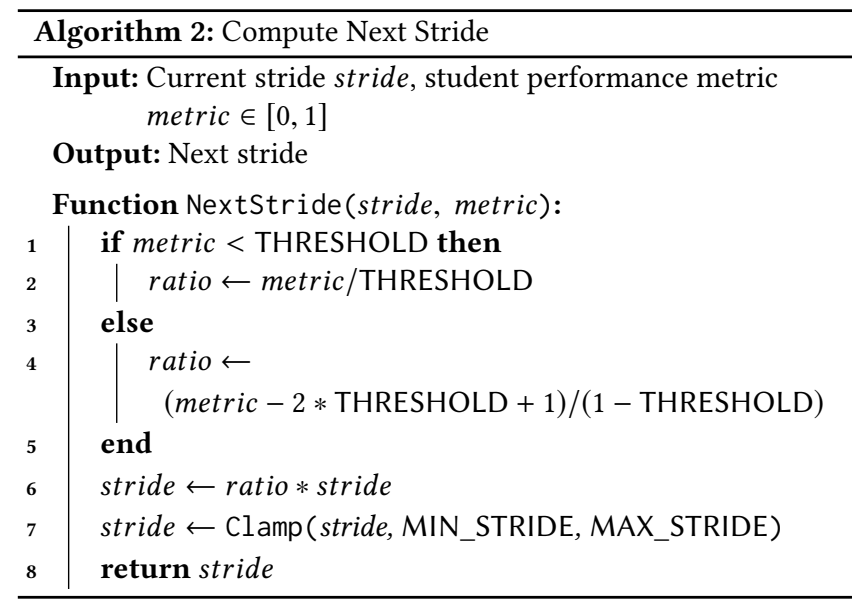

runtime, and student pre-training is done exactly once when the system is first organized.

4.1.5 Key frame striding. After training the student on the current key frame, the distance to the next key frame must be determined. It is logical to change the stride, which defines how often to run student training, based on the student's current performance. However, existing literature regarding key frame striding provides no suitable solution since they are either not adaptive or simplistic (fixed stride [32], exponential back-off [20]), or overly complex (clockwork networks [26], LSTM [2]) for mobile devices. Thus, we aim design a key frame scheduling algorithm that is sufficiently simple but still adaptive, which is presented in 2. Here, the ratio of the next stride to the current one is first determined. Line 2 is a linear function of metric that connects points $(0,0)$ and (THRESHOLD, 1$)$, and line 4 another linear function connecting (THRESHOLD, 1) and (1, 2). Hence, if the student performs better than THRESHOLD, the distance to the next key frame is elongated, and otherwise shortened. Finally, to prevent the stride from vanishing or diverging, we clamp the stride with MIN_STRIDE and MAX_STRIDE. These two parameters must be selected based on the data at hand. For example, if the video has very low FPS and changes quickly even in a few frames, it would be better to choose small numbers for them. Also, too small values will lead to increased network traffic and deteriorate throughput, while too large values will lead to low student performance. We analytically model network traffic and throughput in terms of these algorithm parameters in section 4.4.

\subsection{Partial Knowledge Distillation}

Instead of changing every parameter of the student, which we call full distillation, we suggest to freeze the front part of the network, and only train the rest. This technique is beneficial in terms of latency, memory consumption, and network traffic. Moreover, it is potentially so for student performance (accuracy).

The reduction in latency and memory consumption is immediate. Consider the process of back propagation. Gradients with respect to trainable (not frozen) parameters are computed via chain rule from the loss to the leaf of the computation graph. Thus, if the front part of a neural network is entirely frozen, gradient computation can stop in the middle of the network. This leads to decreases in the amount of computation and latency. Also, memory need not be allocated for the gradients of frozen parameters.

The reduction in network traffic is also evident. Since both the server and the client agree that only a subset of the student's weights will be adapted, it suffices to communicate only the weights that changed. Sending the student weights already incurs little overhead because the student is small by design. Still, partial distillation reduces this overhead further.

The claim of better student performance is rather subtle. Most neural networks are consisted of a front-end feature extractor, 


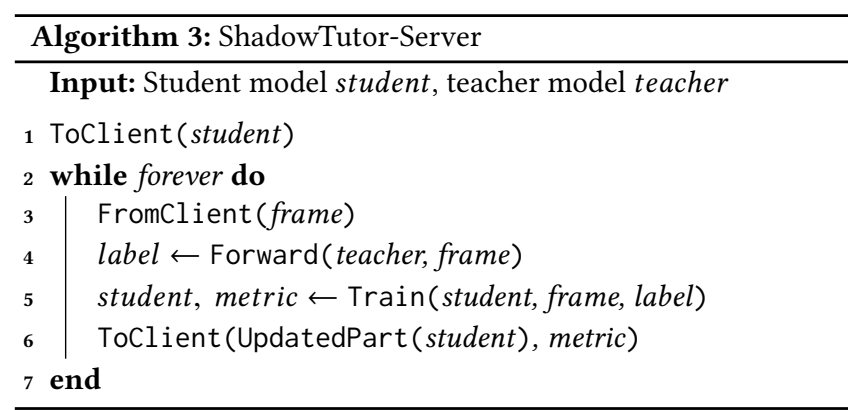

which transforms the input data to a more useful representation, and the back-end network, which maps the representation to a form suitable for the downstream task. Thus, given only a limited number of training steps, jointly training both the feature extractor and the back-end network will be costly and unstable. This is because the front-end network will emit different features every distillation step, and the back-end network must constantly adapt to the change in order to correctly map the feature to the task representation. Thus, adapting only the weights of the back-end network with a fixed feature distribution can be more stable. In a sense, with only a small budget for exploration, it is better to invest on exploitation. Further, drawing reason from existing literature, it is a common practice to freeze the front part of a pre-trained network even for transfer learning [28]. Transfer learning is far more challenging compared with our setting, because it aims to adapt a pre-trained network towards a different task, whereas we stay on the same task.

We empirically validate these claims in section 6 through a series of comparisons with full distillation.

\subsection{ShadowTutor}

Now, we aggregate the system components into ShadowTutor. Algorithm 3 and 4 shows the runtime operations of the server and the mobile device (client) respectively. ShadowTutor offloads teacher inference and student training to the server. Needless to say, teacher inference is an unacceptable workload for mobile devices, so offloading is a natural choice. Student training on mobiles devices is feasible, but it still incurs significant delay. Also, student training blocks student inference; the mobile device cannot proceed with non-key frame inference during student training. This causes large fluctuations in the system's throughput. On the other hand, for the server, training the student only adds marginal overhead since the student only has a small number of parameters.

ShadowTutor reduces network traffic considerably. This is because network communications between the server and the client only occurs at key frames, as opposed to naive offloading, which requires communication for every frame. In addition, thanks to partial distillation, the data size of each network communication is cut down further. Thus, ShadowTutor reduces network traffic in terms of the number of network transmissions and the number of packets in each transmission.

Importantly, offloading student training reveals an opportunity for asynchronous inference. As shown in line 7 and 8 of algorithm 4 , the mobile device sends key frames and receives the updated

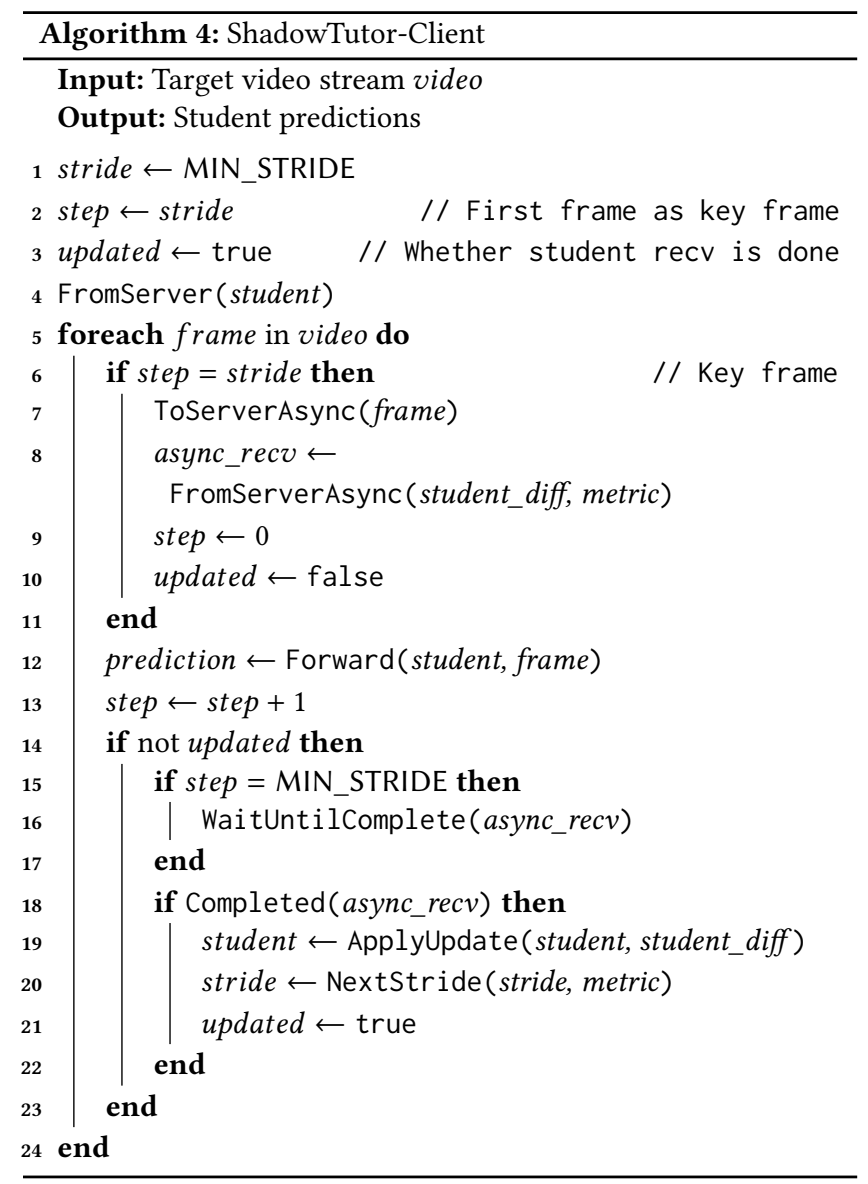

student parameters in a non-blocking fashion. That is, the mobile device sends the key frame to the server, and without waiting for the updated parameters, proceeds on to the next (non-key) frame. This approach further exploits the temporal coherence between frames by assuming that even after a key frame, the student weights are still usable. The updated parameters are awaited for a maximum MIN_STRIDE steps, since the next key frame stride may become MIN_STRIDE. This is the key mechanism that allows ShadowTutor to be robust to adverse network conditions. That is, ShadowTutor can mitigate delays in network transfer by just proceeding further into future frames.

\subsection{Network Traffic and Throughput Bounds}

In this section, we model ShadowTutor's network traffic (amount of data transferred per unit time) and throughput (number of frames processed per unit time) and derive formulae for their lower and upper bounds. We use the notations defined in table 1 . Note that $t_{n e t}$ and $s_{n e t}$ regard the transmission of one key frame and the corresponding updated student parameters.

Both network traffic and throughput rely on the system's total execution time, which we first model. ShadowTutor is designed to perform asynchronous inference for at most MIN_STRIDE many frames after a key frame. However, a mobile device may either be able to execute student inference and network operations entirely in 


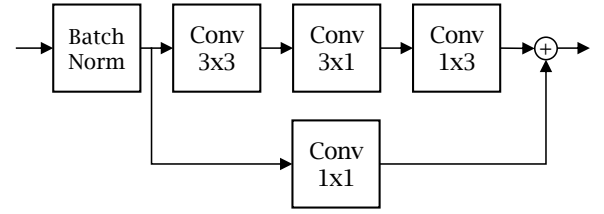

(a) Operations of a student block

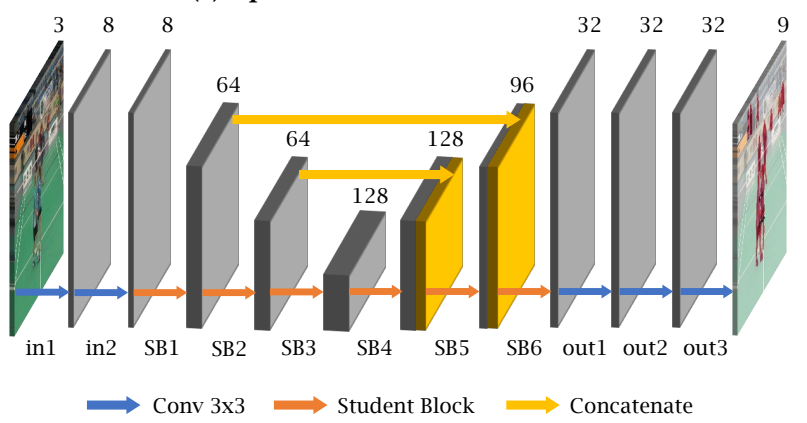

(b) Student architecture

Figure 3: The student model is a small fully-convolutional network.

(pre-trained on the Microsoft COCO dataset [19]) are adopted from the Detectron2 framework.

The student neural network is a simple fully-convolutional network. Figure 3a shows the operations of a student block, and 3b the layer compositions of the student. The low resolution feature maps from SB2 and SB1 are concatenated to the input of SB5 and SB6, respectively. We pre-trained the student on COCO for 30 epochs with the Adam [18] optimizer. As to partial distillation, we freeze the student from the first layer to SB4, only computing gradients until SB5. This way, the trained parameters amount to $21.4 \%$ of all parameters.

Counting the raw number of parameters, the teacher has 44.34 million parameters, whereas the student has 0.48 million. Thus, the teacher is $100 \mathrm{x}$ larger than the student. However, as we will see in section 6.3 , the student can approach the teacher's performance through shadow education.

Model-level optimizations such as using more efficient operations (as in MobileNet [25]) or performing quantization or pruning on weights [11] can be applied to the student. Further, teacher inference can be accelerated with inference optimizations such as TensorRT [22]. However, although these techniques may improve throughput and decrease network traffic, we exclude them in our study. This is because the effect of such techniques differ widely based on implementation, platform, and hardware.

Finally, we decide upon the actual process of knowledge distillation. As pointed out by the LVS dataset paper, videos in the dataset have an excess amount of background class pixels. Due to this class imbalance, training the student with vanilla cross entropy may bias the student towards predicting every pixel as background. Thus, we directly adopt their loss weighting approach, which scales the cross-entropy loss of pixels near and within non-background objects by a factor of 5 . Partial knowledge distillation to the student
Table 2: Execution time and mean number of distillation steps

\begin{tabular}{lll}
\hline Distillation & Partial & Full \\
\hline One step (ms) & 13 & 18 \\
Mean \# of steps & 3.83 & 4.44 \\
\hline
\end{tabular}

parameters is done with the Adam optimizer with a learning rate of 0.01 .

\subsection{Algorithm Parameter Decisions}

We first determine THRESHOLD using the performance of existing video semantic segmentation models. Since the LVS dataset has no official leaderboard available, we consider a similar dataset called Cityscapes [7]. Since the state-of-the-art approach for Cityscapes records an mIOU of 0.845 at the time of research, we set THRESHOLD to 0.8 .

As to MIN_STRIDE and MAX_STRIDE, we consider the FPS of the videos. We have selected videos with an FPS of 25-30. Following this, we set MIN_STRIDE to 8 and MAX_STRIDE to 64 . That is, within 8 frames, we postulate that the objects in the scene will have hardly changed, and re-training within 8 frames would be meaningless. On the other hand, after 64 frames or 2.5 seconds in a video clip, we assume that the objects will have moved significantly, and the student at least needs to be tested.

Finally, we determine MAX_UPDATES using the throughput bounds derived in equation 14 and 15 . Using the notations defined in table 1 , our experiment setting gives $t_{s i}=0.143, t_{s d}=0.013, t_{t i}=0.044$, and $t_{n e t}=0.303$ in seconds. Then, equation 15 yields a maximum throughput of 6.99 FPS. In order to keep the difference between the theoretical maximum and minimum throughput within 2 FPS, we find the largest MAX_UPDATES value that gives a throughput lower bound larger than 5 FPS, which is 8 .

\section{EVALUATION}

Using the configurations from section 5, we show ShadowTutor's effectiveness. Specifically, we investigate the advantages of ShadowTutor in terms of throughput, network traffic, and accuracy in sections 6.1, 6.2, and 6.3, respectively. Then, we push ShadowTutor to the limits in terms of network bandwidth in section 6.4 and temporal coherence in section 6.5.

ShadowTutor is mainly compared with naive offloading (sending every frame to the server and retrieving inference results). Also, we compare partial distillation and full distillation to justify the design of ShadowTutor. All experiments are performed on the first 5000 frames of each video stream (about 200 seconds). Every ShadowTutor experiment, whether partial or full distillation, begin from the same pre-trained student checkpoint.

In or experiments, we only employ one type of teacher model: the Mask R-CNN. This is because the student, who learns from the teacher, is only interested in the final output of the teacher, regardless of all the intermediate operations. 
Table 3: Frames processed per second (FPS) and execution time (s) in parenthesis

\begin{tabular}{lllll}
\hline Camera & Scene & Partial & Full & Naive \\
\hline fixed & animals & $6.55(762.5)$ & $6.21(804.5)$ & $2.09(2391.3)$ \\
fixed & people & $\mathbf{6 . 6 0 ( 7 5 7 . 4 )}$ & $6.43(777.0)$ & $2.09(2391.3)$ \\
fixed & street & $6.50(768.8)$ & $5.95(840.5)$ & $2.09(2391.3)$ \\
moving & animals & $6.57(760.5)$ & $6.27(796.5)$ & $2.09(2391.3)$ \\
moving & people & $\mathbf{6 . 5 9 ( 7 5 8 . 5 )}$ & $6.36(785.8)$ & $2.09(2391.3)$ \\
moving & street & $6.41(780.2)$ & $5.55(901.0)$ & $2.09(2391.3)$ \\
egocentric & people & $6.57(760.5)$ & $5.89(848.5)$ & $2.09(2391.3)$ \\
\hline \multicolumn{2}{c}{ average } & $\mathbf{6 . 5 4 ( 7 6 4 . 1 )}$ & $6.08(822.0)$ & $2.09(2391.3)$ \\
\hline
\end{tabular}

Table 4: Data transmitted on each key frame (MB).

\begin{tabular}{llll}
\hline Direction & Partial & Full & Naive \\
\hline To Server & 2.637 & 2.637 & 2.637 \\
To Client & $\mathbf{0 . 3 9 5}$ & 1.846 & 0.879 \\
\hline Total & $\mathbf{3 . 0 3 2}$ & 4.483 & 3.516 \\
\hline
\end{tabular}

Table 5: Key frames ratio (\%) and network traffic (Mbps)

\begin{tabular}{llllllll}
\hline \multirow{2}{*}{ Camera } & \multirow{2}{*}{ Scene } & \multicolumn{3}{c}{ Key frame ratio } & & \multicolumn{2}{c}{ Network traffic } \\
\cline { 3 - 4 } \cline { 8 - 9 } & & Partial & Full & Naive & & Partial & Naive \\
\hline fixed & animals & 4.73 & 4.60 & 100.0 & & 7.51 & 58.51 \\
fixed & people & $\mathbf{1 . 9 6}$ & 2.42 & 100.0 & & $\mathbf{3 . 1 4}$ & 58.51 \\
fixed & street & 7.78 & 7.43 & 100.0 & & 12.27 & 58.51 \\
moving & animals & 2.55 & 2.29 & 100.0 & & 4.06 & 58.51 \\
moving & people & $\mathbf{3 . 4 5}$ & 4.12 & 100.0 & & 5.51 & 58.51 \\
moving & street & 11.70 & 11.48 & 100.0 & & 18.19 & 58.51 \\
egocentric & people & $\mathbf{5 . 4 6}$ & 9.75 & 100.0 & & 8.70 & 58.51 \\
\hline \multicolumn{2}{r}{ average } & $\mathbf{5 . 3 8}$ & 6.01 & 100.0 & $\mathbf{6 . 1 9}$ & 58.51 \\
\hline
\end{tabular}

\subsection{Throughput}

Table 2 summarizes the latency of one distillation step (ms) and the mean number of distillation steps for partial and full distillation. Partial distillation reduces both the latency and the number of distills, contributing to the throughput of the entire system. This result empirically supports the claim that since the number of training steps is limited, it is quicker to exploit a fixed distribution of features than to explore for better ones.

Table 3 lists the actual throughput of the system (frames processed per second) and the total execution time. As expected, partial distillation outperforms full distillation in every category. Moreover, ShadowTutor shows an improvement greater than $3 x$ over naive offloading. This is especially because ShadowTutor only communicates with the server on key frames, and thus drastically reduces the latency for networking.
Table 6: Mean IoU of various settings. Wild = pre-trained student on its own, $P$ = partial distillation, $F=$ full distillation, digit $(1$ or 8$)=$ number of delayed frames before receiving updated student weights.

\begin{tabular}{|c|c|c|c|c|c|c|}
\hline Camera & Scene & Wild & P-1 & P-8 & F-1 & Naive \\
\hline fixed & animals & 14.34 & 74.31 & 73.27 & 74.47 & 100.0 \\
\hline fixed & people & 13.91 & 81.69 & 81.39 & 81.36 & 100.0 \\
\hline fixed & street & 17.28 & 70.26 & 69.01 & 63.60 & 100.0 \\
\hline moving & animals & 22.31 & 74.94 & 73.80 & 75.21 & 100.0 \\
\hline moving & people & 17.62 & 74.82 & 74.06 & 75.55 & 100.0 \\
\hline moving & street & 18.65 & 60.48 & 58.61 & 52.94 & 100.0 \\
\hline egocentric & people & 14.80 & 70.42 & 68.87 & 61.41 & 100.0 \\
\hline \multicolumn{2}{|c|}{ average } & 16.99 & 72.42 & 71.29 & 69.22 & 100.0 \\
\hline
\end{tabular}

\subsection{Network Traffic}

We investigate the reduction of network traffic in terms of the amount of data transfer per key frame and the ratio of key frames to all frames.

Table 4 shows the amount of data transfer (in MB) per key frame. Since it suffices to send only the updated part of the student, partial distillation reduces network traffic compared with full distillation. Against naive offloading, which sends the teacher prediction to the client, ShadowTutor reduces the amount of data transfer by $13.77 \%$ per key frame, because the size of the student is even smaller than one video frame.

Table 5 summarizes the proportion of key frames and the actual network traffic in Mbps. The smaller the key frame proportion, the less frequent the network communication. Partial distillation generally performs better than full distillation, and strictly better than naive offloading. Especially, for the fixed-people category, the number of network communications is only $1.96 \%$ compared with the naive offloading scheme, yielding a surprising $98 \%$ reduction.

The effects of the two reductions are multiplicative. Thus, compared with naive offloading, ShadowTutor reduces the amount of network transfer per frame by $98.3 \%$ at most, and $95.3 \%$ on average.

On the other hand, the reduction in network traffic (amount of networked data per unit time) is coupled with the improvement in throughput, showing a reduction of $89.4 \%$ on average. We especially note that network traffic has improved even if throughput had a threefold improvement.

Finally, with the current configuration, the network traffic bounds computed with equations 8 and 12 are $2.53 \mathrm{Mbps}$ and $21.2 \mathrm{Mbps}$, respectively. From table 5, all network traffic values obey the bounds, and the bounds are quite tight, proving their usefulness.

\subsection{Accuracy}

Table 6 shows the mean Intersection over Union (mIoU) of various experiment settings. The mIoU of every frame (key and non-key frames) is averaged to show that the student can leverage temporal coherence to accurately perform inference on non-key frames. Note that all accuracy values are evaluated against the teacher (Mask $\mathrm{R}-\mathrm{CNN}$ ) output, which is why the naive approach always achieves perfect accuracy. However, we emphasize that the LVS dataset has 


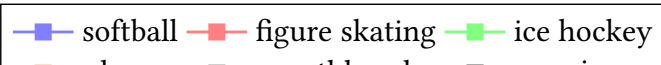
drone - southbeach - naive

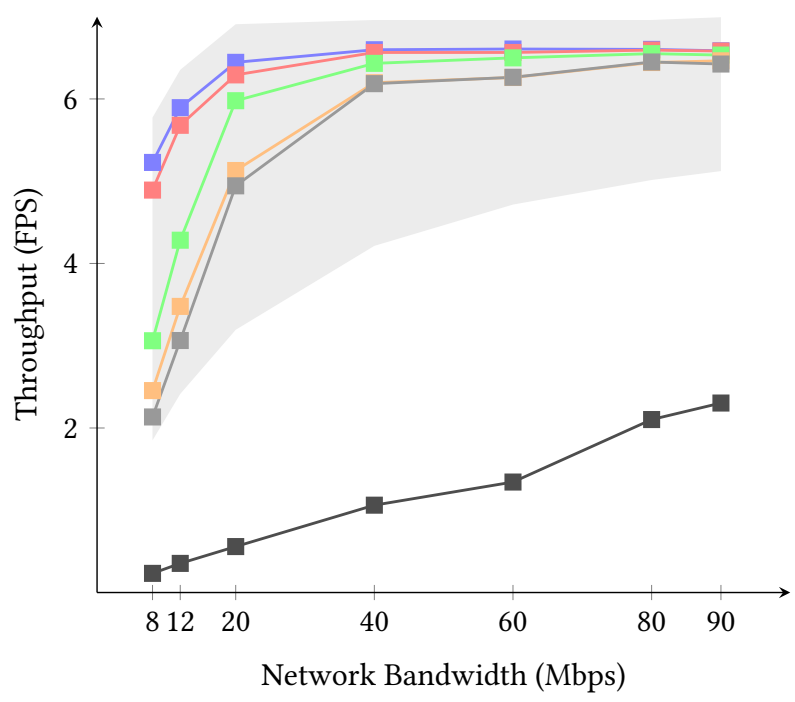

Figure 4: Network bandwidth and system throughput

been labelled with the Mask R-CNN. Thus, in our case, we are measuring the accuracy against the label in effect.

First, to show the need for shadow education, we run the pretrained student on every frame without any supervision from the teacher (denoted as Wild). As expected, its accuracy suffers greatly, approaching the accuracy of random guessing. This is because the student is too small to generalize to all kinds of scenes.

Next, we show the effect of shadow education. Recall that the mobile device receives the updated weights in a non-blocking fashion, mitigating the effect of delays in network transfer. Thus, we measure accuracy when there is the least delay (1 frame, P-1) and the most (8 frames, P-8). ShadowTutor approaches the accuracy of the teacher with a student $100 \mathrm{x}$ smaller, proving the effectiveness of knowledge distillation. Moreover, asynchronous inference hardly hurts accuracy, showing that slightly outdated weights are still useful due to temporal coherence.

Lastly, we compare partial distillation (P-1) and full distillation (F-1). Overall, partial distillation is more accurate. When partial distillation is better, it outperforms full distillation significantly, thereby providing an overall stable level of accuracy.

\subsection{Robustness to Network Conditions}

Fluctuations often happen during network communications between the cloud data center and the client. Thus, in this experiment, we investigate the effect of reduced available network bandwidth. Specifically, we set the bandwidth of the system to 90, 80, 60, 40, 20,12 , and $8 \mathrm{Mbps}$, and examine the throughput of the system.

Figure 4 shows the change in throughput against network bandwidth for ShadowTutor and naive offloading. For ShadowTutor, we selected five video streams with different key frame proportions;
Table 7: Mean IoU and key frame ratio for 7 FPS videos. Digit (1 or 8) = number of frame delays before receiving updated student weights. Key frame proportion is in \%.

\begin{tabular}{|c|c|c|c|c|}
\hline Camera & Scene & Partial-1 & Partial-8 & Key frame \\
\hline fixed & animals & 62.72 & 61.86 & 6.59 \\
\hline fixed & people & 80.44 & 80.08 & 1.97 \\
\hline fixed & street & 63.78 & 62.51 & 8.9 \\
\hline moving & animals & 68.63 & 66.78 & 4.84 \\
\hline moving & people & 73.66 & 72.91 & 4.15 \\
\hline moving & street & 48.92 & 46.99 & 12.34 \\
\hline egocentric & people & 67.57 & 66.09 & 5.44 \\
\hline \multicolumn{2}{|c|}{ average } & 66.53 & 65.31 & 6.32 \\
\hline
\end{tabular}

softball has the least key frames (1.72\%), and southbeach (street CCTV) has the most (12.4\%).

The throughput of naive offloading decreases immediately in the face of low network bandwidth because it has no mechanism to mitigate the increase in network latency. On the contrary, the throughput of ShadowTutor remains remarkably stable until 40 Mbps, which is half of the original bandwidth. For videos that have a small proportion of key frames, throughput is retained even until $20 \mathrm{Mbps}$, since network latency takes up only a small fraction among all latency components. Videos with more key frames lose throughput more quickly, but only by $3 \mathrm{x}$ even if the network bandwidth is $10 \mathrm{x}$ narrower.

The region colored in gray represent the throughput bounds computed with equations 14 and 15. All throughput values obey the bounds. Especially, for low bandwidth settings, network latency dominates among all latency components, reducing the variation in throughput brought about by the degree of concurrency supported by the mobile device.

ShadowTutor's robustness to the reduction in network bandwidth comes from asynchronous inference. In effect, as long as the network latency is shorter than the inference latency of MIN_STRIDE many frames, ShadowTutor can hide the network latency almost completely. However, when the network latency is longer, the reduction in network bandwidth begins to take a more direct impact to the system's throughput since asynchronous inference can no longer serve as a buffer.

\subsection{Feasibility of Real-Time Inference}

Finding promise from 25-30 FPS videos, we test ShadowTutor with videos with less temporal coherence. Specifically, for every video, we re-sample the frames such that all videos have an FPS of 7. Thus, by matching the input video's frame rate with ShadowTutor's throughput, we simulate the real-time inference of frames fetched from the mobile device's camera.

Table 7 shows the mean IoU and key frame proportion of the first 5000 frames of the re-sampled videos. Surprisingly, even if the time distance between adjacent frames is elongated by four times, ShadowTutor yields an average accuracy drop of less than 6\%p and key frame proportion increase of less than $1 \%$ p. Therefore, this shows the feasibility of applying ShadowTutor to real-time inference applications. With optimized students, e.g. those that utilize 
weight quantization/pruning or employ more efficient operations, ShadowTutor will be able to handle higher frame rate videos in real-time, and its accuracy will increase further due to stronger temporal coherence.

\section{RELATED WORK}

We briefly survey works related to extending or replacing parts of ShadowTutor.

The concept of knowledge distillation has been applied to computer vision tasks widely. Chen et al. [5] applied knowledge distillation to creating efficient object detection models for images. Mullapudi et al. [20] extended knowledge distillation to videos. Bajestani et al. [2] extends the previous work by designing an LSTM-based key frame selection method and a teacher-bounded loss function. These works do not consider the context of mobile or distributed computing. Still, they provide insight into knowledge distillation and the possibility of direct application to ShadowTutor.

We may delve further into the possibility of extending ShadowTutor's knowledge distillation process. The original knowledge distillation paper [15] also proposed to distill knowledge from an ensemble of different teacher models. Moreover, data distillation [24] proposes to use only a single teacher, but to ensemble its outputs on the same image but with different transformations applied. Chung et al. [6] proposes to apply knowledge distillation at the feature-level through adversarial training.

\section{CONCLUSION AND FUTURE WORK}

In this work, we developed ShadowTutor, a distributed video DNN inference framework that encodes the temporal coherence in the video at hand into the parameters of a small student model through intermittent partial knowledge distillation. Evaluations show its advantages in terms of network traffic, throughput, accuracy, and robustness. Moreover, ShadowTutor achieves this through a simple and elegant split between the server and the client, and without complex DNN partitioning, server-side scheduling, or model-level optimizations tailored to the experiment devices.

While our work focused on developing a distributed framework for video data, ShadowTutor can also be extended to tasks other than video computer vision. That is, there are plenty of sequence data, i.e. a collection of data points that are temporally coherent, that are handled with DNNs. Examples of such sequence data include speech signals from a single speaker, a sentence that requires translation, or a series of item recommendation requests from a single user. Thus, by exploiting the temporal coherence embedded in various types of sequence data through intermittent knowledge distillation, ShadowTutor has the potential to be extended to any type of sequence data. Upgrading the knowledge distillation process, designing appropriate model architectures for the teacher and the student, and resolving the issues that may arise in the process will serve as a good future research direction.

\section{ACKNOWLEDGMENTS}

This work was supported by the National Research Foundation of Korea (NRF) grant funded by the Korea government (MSIT) (No. 2020R1A2B5B02001845).

\section{REFERENCES}

[1] G. Ananthanarayanan, P. Bahl, P. Bodík, K. Chintalapudi, M. Philipose, L. Ravindranath, and S. Sinha. 2017. Real-Time Video Analytics: The Killer App for Edge Computing. Computer 50, 10 (2017), 58-67.

[2] M. F. Bajestani and Y. Yang. 2020. TKD: Temporal Knowledge Distillation for Active Perception. In The IEEE Winter Conference on Applications of Computer Vision. 953-962.

[3] V. Bazarevsky and A. Tkachenka. 2018. Mobile Real-Time Semantic Segmentation. Retrieved March 13, 2020 from https://ai.googleblog.com/2018/03/mobile-realtime-video-segmentation.html

[4] W. Chan, N. Jaitly, Q. Le, and O. Vinyals. 2016. Listen, attend and spell: A neural network for large vocabulary conversational speech recognition. In 2016 IEEE International Conference on Acoustics, Speech and Signal Processing (ICASSP). 4960-4964.

[5] G. Chen, W. Choi, X. Yu, T. Han, and M. Chandraker. 2017. Learning efficient object detection models with knowledge distillation. In Advances in Neural Information Processing Systems. 742-751.

[6] I. Chung, S. Park, J. Kim, and N. Kwak. 2020. Feature-map-level Online Adversarial Knowledge Distillation. arXiv preprint arXiv:2002.01775 (2020).

[7] M. Cordts, M. Omran, S. Ramos, T. Rehfeld, M. Enzweiler, R. Benenson, U. Franke, S. Roth, and B. Schiele. 2016. The Cityscapes Dataset for Semantic Urban Scene Understanding. In Proc. of the IEEE Conference on Computer Vision and Pattern Recognition (CVPR).

[8] A. Eshratifar and M. Pedram. 2018. Energy and performance efficient computation offloading for deep neural networks in a mobile cloud computing environment. In Proceedings of the 2018 on Great Lakes Symposium on VLSI. 111-116.

[9] Andrew G. 2019. Mobile GPU approaches to power efficiency. Retrieved March 3, 2020 from https://www.highperformancegraphics.org/wp-content/uploads/2019/ hot3d/mobile_gpu_power_and_performance.pdf

[10] E. Gabriel, G. Fagg, G. Bosilca, T. Angskun, J. Dongarra, J. Squyres, V. Sahay, P. Kambadur, B. Barrett, A. Lumsdaine, R. Castain, D. Daniel, R. Graham, and T. Woodall. 2004. Open MPI: Goals, Concept, and Design of a Next Generation MPI Implementation. In Proceedings, 11th European PVM/MPI Users' Group Meeting. 97-104.

[11] S. Han, H. Mao, and W. Dally. 2015. Deep compression: Compressing deep neural networks with pruning, trained quantization and huffman coding. arXiv preprint arXiv:1510.00149 (2015)

[12] S. Han, H. Shen, M. Philipose, S. Agarwal, A. Wolman, and A. Krishnamurthy. 2016. MCDNN: An Approximation-Based Execution Framework for Deep Stream Processing Under Resource Constraints. In Proceedings of the 14th Annual International Conference on Mobile Systems, Applications, and Services (MobiSys '16). ACM, 123-136.

[13] K. He, G. Gkioxari, P. Dollár, and R. Girshick. 2017. Mask R-CNN. In 2017 IEEE International Conference on Computer Vision (ICCV). 2980-2988.

[14] B. Hidasi, A. Karatzoglou, L. Baltrunas, and D. Tikk. 2015. Session-based recommendations with recurrent neural networks. (2015). arXiv:1511.06939

[15] G. Hinton, O. Vinyals, and J. Dean. 2015. Distilling the knowledge in a neural network. arXiv preprint arXiv:1503.02531 (2015).

[16] IBM. 2018. Analyze real-time CCTV images with Convolutional Neural Networks. Retrieved March 14, 2020 from https://developer.ibm.com/patterns/iotdevicesensor-damage-detection-with-edge-analytics/

[17] Y. Kang, J. Hauswald, C. Gao, A. Rovinski, T. Mudge, J. Mars, and L. Tang. 2017. Neurosurgeon: Collaborative Intelligence Between the Cloud and Mobile Edge. In Proceedings of the Twenty-Second International Conference on Architectural Support for Programming Languages and Operating Systems (Xi'an, China) (ASPLOS '17). ACM, 615-629.

[18] D. Kingma and J. Ba. 2015. Adam: A Method for Stochastic Optimization. In 3rd International Conference on Learning Representations, ICLR 2015, San Diego, CA, USA, May 7-9, 2015, Conference Track Proceedings, Y. Bengio and Y. LeCun (Eds.).

[19] T. Lin, M. Maire, S. Belongie, J. Hays, P. Perona, D. Ramanan, P. Dollár, and C. L. Zitnick. 2014. Microsoft coco: Common objects in context. In European conference on computer vision. Springer, 740-755.

[20] R. Mullapudi, S. Chen, K. Zhang, D. Ramanan, and K. Fatahalian. 2019. Online model distillation for efficient video inference. In Proceedings of the IEEE International Conference on Computer Vision. 3573-3582.

[21] NVIDIA. 2020. NVIDIA Jetson Nano. https://www.nvidia.com/en-us/ autonomous-machines/embedded-systems/jetson-nano/

[22] NVIDIA. 2020. NVIDIA TensorRT. Retrieved February 28, 2020 from https: //developer.nvidia.com/tensorrt

[23] A. Paszke, S. Gross, F. Massa, A. Lerer, J. Bradbury, G. Chanan, T. Killeen, Z. Lin, N. Gimelshein, L. Antiga, A. Desmaison, A. Kopf, E. Yang, Z. DeVito, M. Raison, A. Tejani, S. Chilamkurthy, B. Steiner, L. Fang, J. Bai, and S. Chintala. 2019. PyTorch: An Imperative Style, High-Performance Deep Learning Library. In Advances in Neural Information Processing Systems 32, H. Wallach, H. Larochelle, A. Beygelzimer, F. d'Alché-Buc, E. Fox, and R. Garnett (Eds.). Curran Associates, Inc., 8024-8035. 
[24] I. Radosavovic, P. Dollár, R. Girshick, G. Gkioxari, and K. He. 2018. Data distillation: Towards omni-supervised learning. In Proceedings of the IEEE Conference on Computer Vision and Pattern Recognition. 4119-4128.

[25] M. Sandler, A. Howard, M. Zhu, A. Zhmoginov, and L. Chen. 2018. Mobilenetv2 Inverted residuals and linear bottlenecks. In Proceedings of the IEEE conference on computer vision and pattern recognition. 4510-4520.

[26] E. Shelhamer, K. Rakelly, J. Hoffman, and T. Darrell. 2016. Clockwork convnets for video semantic segmentation. In European Conference on Computer Vision. Springer, 852-868.

[27] M. Siam, S. Elkerdawy, M. Jagersand, and S. Yogamani. 2017. Deep semantic segmentation for automated driving: Taxonomy, roadmap and challenges. In 2017 IEEE 20th international conference on intelligent transportation systems (ITSC). IEEE, $1-8$.

[28] C. Tan, F. Sun, T. Kong, W. Zhang, C. Yang, and C. Liu. 2018. A survey on deep transfer learning. In International conference on artificial neural networks. Springer,
$270-279$.

[29] A. Vaswani, N. Shazeer, N. Parmar, J. Uszkoreit, L. Jones, A. Gomez, L. Kaiser, and I. Polosukhin. 2017. Attention is All You Need. In Proceedings of the 31st International Conference on Neural Information Processing Systems (Long Beach, California, USA) (NIPS'17). Curran Associates Inc., Red Hook, NY, USA, 6000-6010.

[30] Y. Wu, A. Kirillov, F. Massa, W. Lo, and R. Girshick. 2019. Detectron2. https: //github.com/facebookresearch/detectron2.

[31] H. Yang, T. Fu, P. Chiang, K. Ho, and C. Lee. 2019. A Distributed Scheme for Accelerating Semantic Video Segmentation on An Embedded Cluster. In 2019 IEEE 37th International Conference on Computer Design (ICCD). 73-81.

[32] X. Zhu, Y. Xiong, J. Dai, L. Yuan, and Y. Wei. 2017. Deep feature flow for video recognition. In Proceedings of the IEEE Conference on Computer Vision and Pattern Recognition. 2349-2358. 